\title{
Membrane disruption by optically controlled microbubble cavitation
}

\author{
PAUL PRENTICE ${ }^{1}$, ALFRED CUSCHIERI ${ }^{1}$, KISHAN DHOLAKIA ${ }^{2}$, MARK PRAUSNITZ ${ }^{3}$ AND \\ PAUL CAMPBELL $1,4 *$
}

${ }^{1}$ Institute of Medical Science and Technology, Department of Surgery and Molecular Oncology, Ninewells Hospital, University of Dundee, Dundee DD1 9SY, Scotland, UK ${ }^{2}$ School of Physics and Astronomy, University of St Andrews, North Haugh, St Andrews KY16 9SS, Fife, Scotland, UK

${ }^{3}$ School of Chemical and Biomolecular Engineering, Georgia Institute of Technology, 311 Ferst Drive, Atlanta, Georgia 30332-0100, USA

${ }^{4}$ Div. Electronic Engineering and Physics, University of Dundee, Dundee DD1 4HN, Scotland, UK

*e-mail: p.a.campbell@dundee.ac.uk

n fluids, pressure-driven cavitation bubbles have a nonlinear response that can lead to extremely high core-energy densities during the collapse phase-a process underpinning phenomena such as sonoluminescence ${ }^{1}$ and plasma formation ${ }^{2}$. If cavitation occurs near a rigid surface, the bubbles tend to collapse asymmetrically, often forming fast-moving liquid jets that may create localized surface damage ${ }^{3}$. As encapsulated microbubbles are commonly used to improve echo generation in diagnostic ultrasound imaging, it is possible that such cavitation could also lead to jet-induced tissue damage. Certainly ultrasonic irradiation (insonation) of cells in the presence of microbubbles can lead to enhanced membrane permeabilization and molecular uptake (sonoporation) $)^{4-7}$, but, although the mechanism during low-intensity insonation is clear ${ }^{8}$, experimental corroboration for higher pressure regimes has remained elusive. Here we show direct observational evidence that illuminates the energetic micrometre-scale interactions between individual cells and violently cavitating shelled microbubbles. Our data suggest that sonoporation at higher intensities may arise through a synergistic interplay involving several distinct processes.

Selective introduction of therapeutic agents to the interior of cells remains a challenging problem, principally because the lipid membrane acts as an impermeable barrier to most water-soluble molecules. To address this issue, physical approaches are attractive as they typically offer more generic applicability compared with viral or biochemical counterparts. Of the approaches devised thus far, both electroporation ${ }^{9}$ and photoporation ${ }^{10}$ have demonstrated impressive in vitro results. However, their development for use in general (non-topical) clinical settings would typically require an invasive procedure. In contrast, using ultrasound to mediate molecular delivery to remote anatomical sites has exciting potential as it may be applied extracorporeally, and may also be focused to specific localized regions internally.

Preliminary in vitro observations using ultrasound in the megahertz range at higher pressures $(>0.2 \mathrm{MPa})$ have underscored this promise ${ }^{4-6}$ and, significantly, tumour regression has also
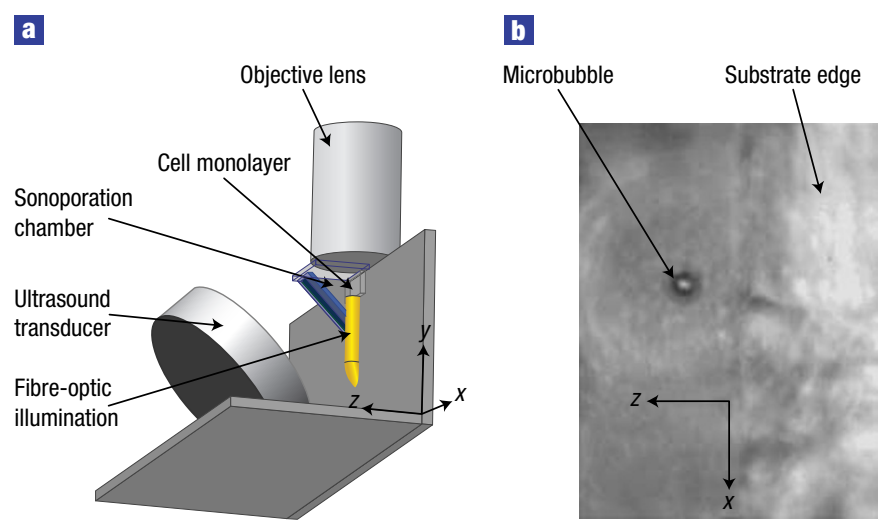

Figure 1 Apparatus and configuration of the optically trapped microbubble in relation to a coverslip/monolayer system. a, For orientational purposes, we designate the substrate surface to occupy the $x y$ plane with the trapped microbubble displaced in the orthogonal $z$ direction. $A \times 60$ objective lens $(\mathrm{NA}=0.9)$ acts as both the focusing lens for the laser-trapping beam and also constitutes our principal magnification element. $\mathbf{b}$, The view obtained through the objective lens showing a trapped microbubble held at a controlled displacement from the coverslip.

been demonstrated in murine studies ${ }^{7}$. However, categorical identification of possible membrane-disruption mechanisms in this clinically important regime of higher mechanical index have remained elusive. Definitive confirmation requires direct imaging of the actual precursor events that disrupt the membrane followed by correlation with any induced membrane features. The results presented here realize both these critical objectives. Moreover, we wished to ascertain the disruptive potential of ultrasoundstimulated microbubbles in the most general context, namely when they are in the vicinity of, but not directly adhered 

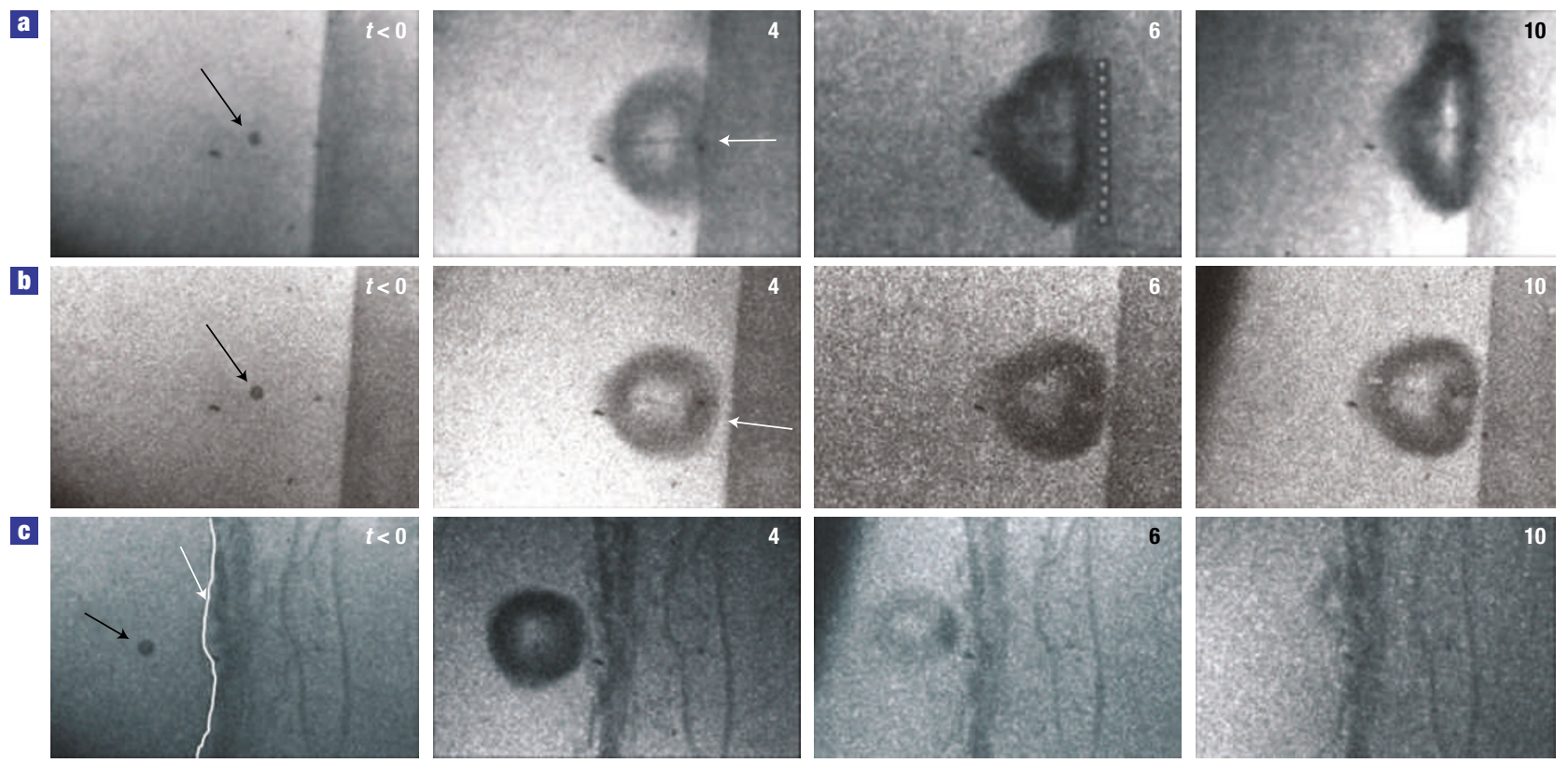

d

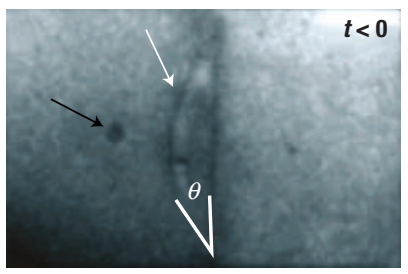

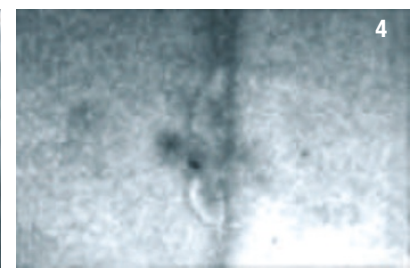
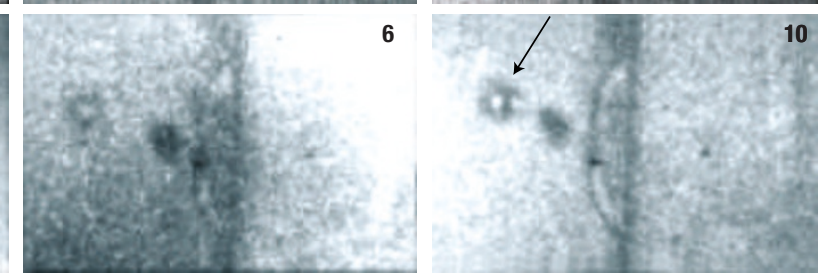

Figure 2 Representative UHS sequences acquired at a framing rate of $500 \mathrm{kHz}$ showing microbubble cavitation, in proximity to naked coverslips (a, b) and at cell monolayers (c, d). Timings (in microseconds) relative to the instant of cavitation inception are indicated on each frame. Images were spatially calibrated by observing $6 \mu \mathrm{m}$ calibration beads ( 12 such beads have been superimposed on the scene to provide scale in the third frame of sequence a). Each frame measures $163 \mu \mathrm{m} \times 110 \mu \mathrm{m}$. a, Initially $(t<0)$ a $4.5-\mu \mathrm{m}$-diameter microbubble (arrowed black throughout) was trapped and manipulated to a displacement $26.5 \mu \mathrm{m}$ from the substrate (darker region to the right) before ultrasonic stimulation to produce jets (arrowed white throughout) (see also Supplementary Information, Movie S1). b, By controlled displacement of a similarly sized UCA to a location some $19 \mu \mathrm{m}$ farther from the substrate and then insonating, only the microjet is seen to touch down at the surface (at $6 \mu \mathrm{s}$ ) (see Supplementary Information, Movie S2). c, The dominant event on cells (white arrow with membrane highlighted in white also), was quasispherical expansion of microbubbles into osculation with the underlying monolayer (see Supplementary Information, Movie S3). d, Sonic cracking in proximity to a cell, and reformation of the gas core as a free bubble (black arrow, rightmost frame) (see Supplementary Information, Movie S4).

to, cells. This requires the experimental capability to spatially control the microbubble position relative to adjacent surfaces. Previous methods for positioning cavitation nuclei accurately, such as laser-induced bubble formation or resonant positioning by Bjerknes forces, are respectively inapplicable or restrictive in our experimental architecture (Fig. 1). Instead, we controlled the individual ultrasound-contrast-agent (UCA) microbubbles by tweezing them in Laguerre-Gauss optical traps ${ }^{11}$. To retain clinical relevance, we used commercial encapsulated Optison UCAs throughout (complete system details and methodology can be found in the accompanying Supplementary Information). Once tweezed, the UCA microbubbles were guided to predefined $z$ displacements (Fig. 1b) from a substrate plane and an insonation protocol applied (typically a $20-\mu$ s burst of $1-\mathrm{MHz}$ ultrasound at peak negative pressure (p.n.p.) $=1.39 \mathrm{MPa}( \pm 14 \%))$, with synchronous triggering of an ultra-high-speed (UHS) camera. More than 250 UHS sequences were acquired; however, for the present analysis we only considered those events showing bubble radii at maximum expansion, $R_{\mathrm{MAX}} \geq z_{0}$, where $z_{0}$ represents the quiescent UCA-to-substrate displacement.

Individual trapped UCAs that were insonated in bulk solution (deionized water) underwent spherically symmetric expansion and collapse. However, when UCAs were trapped close to a rigid substrate, a significant departure from symmetric behaviour typically occurred. For insonation pressures with p.n.p. $\geq 0.5 \mathrm{MPa}$, then on cavitation inception the UCAs were typically seen to expand rapidly, with spontaneous development of a thin micrometre-width filament that traversed the bubble volume in the direction orthogonal to the substrate plane (Fig. 2a). Such features are indicative of involuted fluid microjets, and seem morphologically similar to previous UHS observations of inertial cavitation in millimetre-scale unshelled gas bubbles ${ }^{3,12}$. The observations also confirm theoretical predictions that such entities are entirely feasible on the micrometre scale ${ }^{13}$. Indeed, a form of jetting behaviour has been previously noticed in micrometresized free (that is, unshelled) bubbles exposed to extremely high pressure $(11 \leq$ p.n.p. $\leq 100 \mathrm{MPa})$ shock waves ${ }^{14}$. However, it is clear from the present observations that 'microjetting' can also arise in encapsulated bubbles, and at much reduced pressure amplitudes, when cavitation occurs in proximity to rigid substrates.

Once $R_{\mathrm{MAX}}$ for a specific ultrasound pressure amplitude was measured, we exploited the spatial control afforded by our apparatus to refine the interaction of a microbubble with the substrate. By retracting a bubble to a displacement just beyond its 


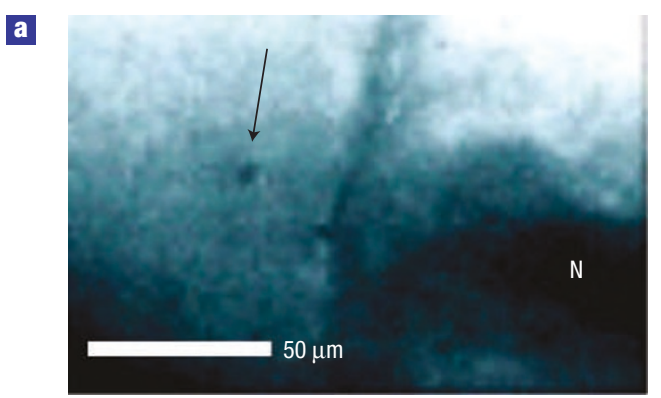

b
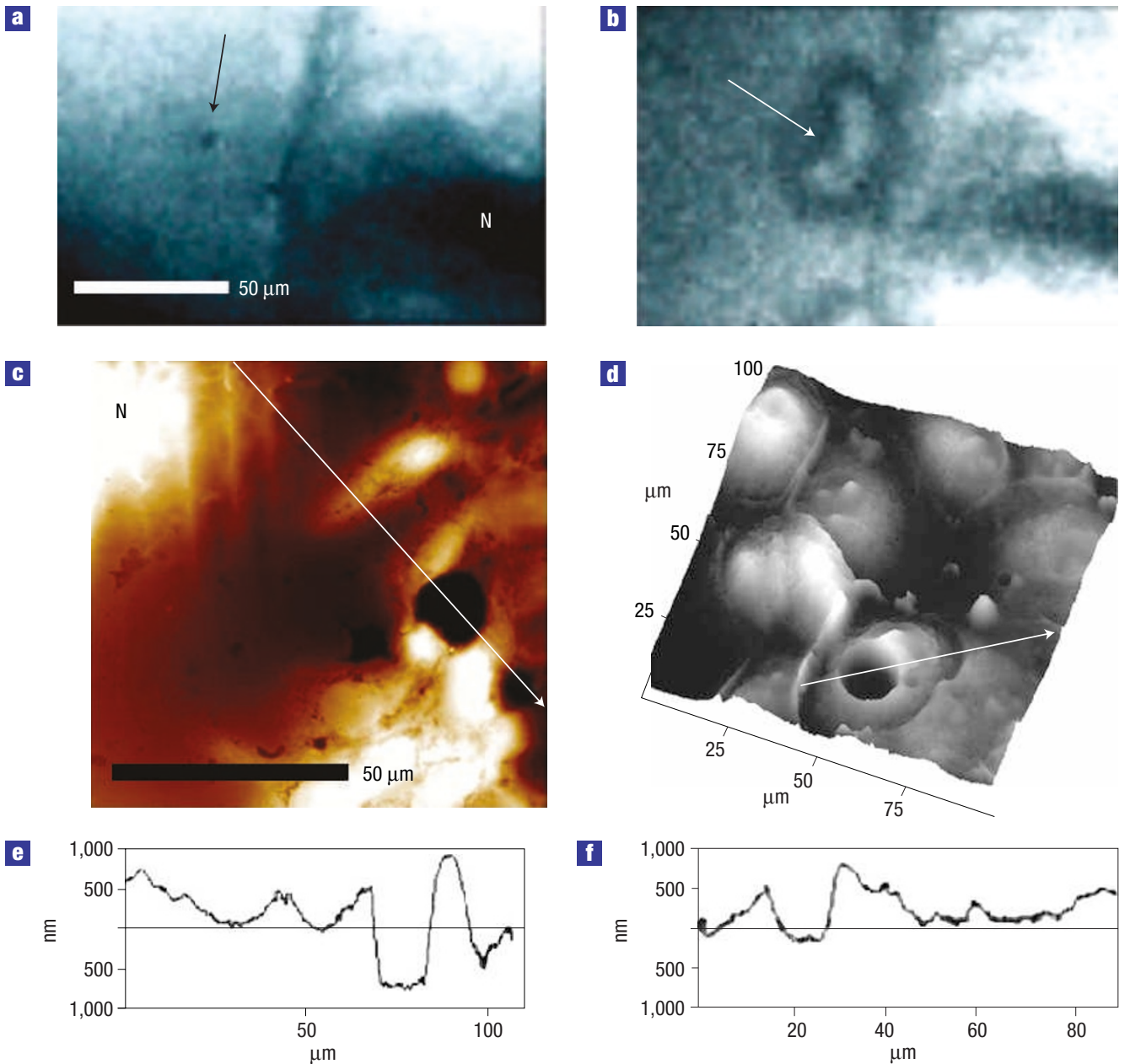

f

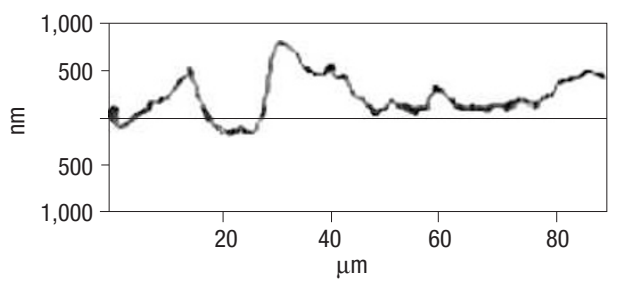

Figure 3 Correlation of specific cavitation events with membrane damage. a, A $163 \mu \mathrm{m} \times 110 \mu \mathrm{m}$ frame showing a quiescent 4- $\mu \mathrm{m}$-diameter microbubble trapped $17 \mu \mathrm{m}$ from a cell membrane (see text for definition of $\mathrm{N}$ ). $\mathbf{b}$, At $t=8 \mu \mathrm{s}$ after cavitation inception, the microbubble has developed an involution (arrowed), which is in contact with the membrane over a region some $15 \mu \mathrm{m}$ wide. Scale bar as for a. c, An AFM image (JPK Instruments NanoWizard) of fixed cells. The resultant sonopore measures $16 \mu \mathrm{m}$, as indicated on the respective topographical cross-section (white arrow) of e. d, A perspective view (taken with a Veeco Bioscope AFM) of another sonopore, the cross-section (white arrow) of which is shown in $\mathbf{f}$.

measured $R_{\mathrm{MAX}}$ and then insonating, microjet 'touchdown' alone could be achieved (Fig. 2b). Microjetting was relatively common on naked substrates, occurring in 39\% (9/23) of this data set. The remaining observations simply involved quasispherical UCA shell expansion into osculation with the substrate, without the formation of any obvious microjet.

Substrates supporting cultured monolayers of adherent live MCF7 (human breast cancer) cells at $70-80 \%$ confluence were introduced to the sonoporation chamber (containing Dulbeccos modified Eagle's medium). On insonation, the dominant finding here again was quasispherical expansion (48\% (11/23)) with shell contact at $R_{\mathrm{MAX}}$ (Fig. 2c). We observed that, for all values of $z_{0} / R_{\mathrm{MAX}} \leq 1$, compression of the underlying cells would also then occur. This process may in itself lead to membrane permeabilization ${ }^{15,16}$. Interestingly, however, another phenomenon, 'sonic-cracking'17,18, was observed in $35 \%$ of data acquired on monolayers $(8 / 23)$. In this instance, the UCA shell ruptures during the initial phase of cavitation, rapidly (within $2 \mu \mathrm{s}$ ) giving rise to an ejection of core gas (perfluorocarbon) consistently directed away from the coverslip plane. To conserve momentum, it seems that the shell remnant is then ballistically propelled towards the monolayer, compressing any intervening cells (Fig. 2d). Calculations suggest that this occurrence could also constitute a viable candidate for membrane permeabilization (see Supplementary Information).

Active microjetting into cells was observed in 17\% (4/23) of this data set. Here, UCAs underwent inertial cavitation and collapse, with the outermost bubble hemisphere undergoing involution to form a central reentrant jet directed at the cell (Fig. 3b). We also noticed a secondary effect during extended UHS imaging periods (up to $62 \mu \mathrm{s}$ ) where the microbubbles would undergo inertial cavitation and jetting, often with a secondary, but much weaker, inflation cycle (see Supplementary Information, Movie S5). To examine the penetrability of UCA microjets, post-insonation membrane topographies were interrogated using atomic force microscopy (AFM). Rapid registration of those specific cells that had been previously addressed by an optically trapped UCA was achieved by notching the substrate edges to create alignment markers. One such notch is manifested by the shadow to the lower right of Fig. 3a (labelled ' $N$ '). This same notch can also be seen in the AFM image (Fig. 3c; top left) as a raised region of the substrate topography. AFM imaging of control cells that had not been insonated showed no evidence of membrane breaching. 
Interestingly, no obvious disruption was observed on cells exposed to 'sonic cracking', even though our calculations suggest that the membrane may be breached by this process. It may be that any induced (small) pores are thermodynamically unstable and were resealed ${ }^{19}$ before downstream chemical fixing occurred. Alternatively, a similarly rapid vesicle-patching response ${ }^{20}$ may have been elicited. Supporting experiments confirming this effective time frame for membrane sealing (several seconds) have been demonstrated by patch-clamp measurements on cells that had been insonated in the presence of Optison ${ }^{21}$.

By contrast, the disruption to cells that had been exposed to microjetting was pronounced. Single deep pits were observed on all such samples. Figure $3 \mathrm{c}$ shows one such pit formed because of the jetting event depicted in Fig. 3a and b. A topographical cross-section through this pit (Fig. 3e) reveals the pit diameter to be approximately $16 \mu \mathrm{m}$ across, similar to that observed during UHS imaging of the microjet 'touchdown' (Fig. 3b). Moreover, the jet penetration power was such that the pit depth extends to the underlying substrate. Figure $3 \mathrm{~d}$ shows a perspective AFM image of a representative 'sonopore' formed on a different cell monolayer and having a distinct peripheral lip of raised $(>500 \mathrm{~nm})$ material (Fig. 3f). The spatial extent of such microjet-induced pits would seem to suggest that lysis is inevitable. Indeed, this echoes the findings of others, that insonation in the presence of Optison with elevated ultrasound pressures is linked to enhanced occurrence of cell death ${ }^{4,5,22}$.

Reconciling our observations against potential microscopic mechanisms is non-trivial, given the slightly restrictive temporal resolution of our UHS sequences. With inter-frame times of the order of a microsecond, only a lower-bound estimate for the jet velocities, $v_{\mathrm{i}}$, can be made, equating to approximately $5.5 \mathrm{~m} \mathrm{~s}^{-1}$ (distance traversed, $22 \mu \mathrm{m}$, divided by traversal time, $4 \mu \mathrm{s}$, in Fig. 3a,b). Membrane breaching is thought possible if any induced tension exceeds the critical rupture stress, $\tau_{\text {crit }}$, which is related to the local elastic modulus, $E$,

$$
\tau_{\text {crit }}=E \cdot \varepsilon_{\mathrm{r}}
$$

Here $\varepsilon_{\mathrm{r}}$ is the relative deformation (critical areal strain) needed for rupture to occur, a value commonly accepted ${ }^{23}$ as $3 \%$; hence $\varepsilon_{\mathrm{r}}=0.03$. Measurements of $E$ on live cells are consistently below $100 \mathrm{kPa}$ (ref. 24) leading to an approximate upper limit of $\tau_{\text {crit }} \approx 3 \mathrm{kPa}$. Consideration of two distinct candidate mechanisms ${ }^{18,25}$, the development of a water hammer pressure, $P_{\mathrm{WH}}$, and momentum exchange because of fluid flow, $P_{\mathrm{F}}$, allows estimates of their respective induced pressures to be ascertained. As $P_{\mathrm{WH}} \approx 0.5 \rho c v_{\mathrm{j}}$, where $\rho$ is the fluid density $\left(998 \mathrm{~kg} \mathrm{~m}^{-3}\right)$ and $c$ is the speed of sound in the medium $\left(1,480 \mathrm{~m} \mathrm{~s}^{-1}\right)$, thus $P_{\mathrm{WH}} \approx 4 \mathrm{MPa}$. Adopting the approach in which the flow-induced pressure at the stagnation point is represented as $0.5 \rho v_{j}^{2}$ (ref. 25), we thus calculate $P_{\mathrm{F}} \approx 15 \mathrm{kPa}$. Therefore, as both pressure estimates exceed $\tau_{\text {crit }}$, either (or both) are then viable breaching mechanisms in this high-mechanical-index regime. Whereas gentler insonation regimes thus seem to induce sonoporation by a dominant bubble-induced shear flow ${ }^{8}$, in more aggressive ultrasound regimes we have observed that several further processes warrant attention as synergistic contributors to the overall state of permeabilization.

Achieving a more complete understanding of the statistics shown by our observations requires further investigation on both the experimental and theoretical fronts. Even though cavitation processes are well known to be stochastic in nature, with the further complication of encapsulated UCAs, intrinsic variations in microbubble shell uniformity may also be a factor, especially for the process of sonic cracking $^{18}$. In the more general case, absolute values for shell rigidity may also be important in dictating the prevalent microbubble behaviour. Coupling a computational fluid-dynamics approach with parallel structural mechanics to accommodate both the UCAs and any nearby walls may prove useful in illuminating the dominant hydrodynamic processes arising. The predictive power of this approach will be especially useful when the code has been validated against controlled UHS observations on shelled UCAs, such as those presented here.

Received 20 April 2005; accepted 22 September 2005; published 23 October 2005.

\section{References}

1. Hilgenfeldt, S., Grossmann, S. \& Lohse, D. A simple explanation of light emission in sonoluminescence. Nature 398, 402-405 (1999).

2. Flannigan, D. J. \& Suslick, K. S. Plasma formation and temperature measurement during single bubble cavitation. Nature 434, 52-55 (2005).

3. Benjamin, T. B. \& Ellis, A. T. The collapse of cavitation bubbles and the pressure thereby produced against solid boundaries. Phil. Trans. R. Soc. Lond. A 260, 221-245 (1966).

4. Ward, M., Wu, J. \& Chiu, J. F. Ultrasound induced cell lysis and sonoporation enhanced by contrast agents. J. Acoust. Soc. Am. 105, 2951-2957 (1999).

5. Feril, L. B. et al. Enhancement of ultrasound-induced apoptosis and cell lysis by echo contrast agents. Ultrasound Med. Biol. 29, 331-337 (2003).

6. Honda, H., Kondo, T., Zhao, Q. L., Feril, L. B. \& Kitagawa, H. Role of intracellular calcium ions and reactive oxygen species in apoptosis induced by ultrasound. Ultrasound Med. Biol. 30, 683-692 (2004).

7. Miller, D. L. \& Song, J. Tumour growth reduction and DNA transfer by cavitation-enhanced high intensity focused ultrasound in vivo. Ultrasound Med. Biol. 29, 887-893 (2003).

8. Marmottant, P. \& Hilgenfeldt, S. Controlled vesicle deformation and lysis by single oscillating bubbles. Nature 423, 153-156 (2003).

9. Canatella, P. J. \& Prausnitz, M. R. Prediction and optimization of gene transfection and drug delivery by electroporation. Gene Therapy 8, 1464-1469 (2001).

10. Tirlapur, U. K. \& König, K. Targeted transfection by femtosecond laser. Nature 418, 290-291 (2002).

11. Gahagan, K. T. \& Swartzlander, G. A. Trapping of low-index microparticles in an optical vortex. J. Opt. Soc. Am. B 15, 524-534 (1998).

12. Phillip, A. \& Lauterborn, W. Cavitation erosion by single laser-produced bubbles. J. Fluid Mech. 361, 75-116 (1998).

13. Plesset, M. S. \& Chapman, R. B. Collapse of an initially spherical vapour cavity in the neighbourhood of a solid boundary. J. Fluid. Mech. 47, 283-290 (1971)

14. Ohl, C. D. \& Ikink, R. Shock wave induced jetting of micron size bubbles. Phys. Rev. Lett. 90, 214502 (2003).

15. Van Wamel, A., Bouakaz, A., Versluis, M. \& de Jong, N. Micromanipulation of endothelial cells: Ultrasound-microbubble-cell interactions. Ultrasound Med. Biol. 30, 1255-1258 (2004).

16. Wolfram, B., Mettin, R., Kurz, T. \& Lauterborn, W. Observations of pressure wave excited contrast agent microbubbles in the vicinity of cells. Appl. Phys. Lett. 81, 5060-5062 (2002).

17. Postema, M., Van Wamel, A., Lancée, C. T. \& de Jong, N. Ultrasound-induced encapsulated microbubble phenomena. Ultrasound Med. Biol. 30, 827-840 (2004).

18. Postema, M. Medical Bubbles (Universal, Veenedaal, Netherlands, 2004).

19. Weaver, J. C. \& Chizmadzev, Y. A. Theory of electroporation: A review. Bioelectrochem. Bioenerget. 41, 135-160 (1996).

20. Steinhardt, R. A., Bi, G. \& Alderton, J. M. Cell membrane resealing by a vesicular mechanism similar to neurotransmitter release. Science 263, 390-393 (1994).

21. Deng, C. X., Sieling, F., Pan, H. \& Cui, J. Ultrasound induced cell membrane porosity. Ultrasound Med. Biol. 30, 519-526 (2004).

22. Guzman, H. R., Nguyen, D. X., Khan, S. \& Prausnitz, M. R. Ultrasound-mediated disruption of cell membranes. II. Heterogeneous effects on cells. J. Acoust. Soc. Am. 110, 597-606 (2001).

23. Boal, D. Mechanics of the Cell (Cambridge Univ. Press, Cambridge, 2002).

24. Rotch, C., Jacobson, K. \& Radmacher, M. Dimensional and mechanical dynamics of active and stable edges in motile fibroblasts investigated using atomic force microscopy. Proc. Natl Acad. Sci. USA 96, 921-926 (1999).

25. Brujan, E. A. The role of cavitation microjets in the therapeutic applications of ultrasound. Ultrasound Med. Biol. 30, 381-387 (2004).

\section{Acknowledgements}

We thank the EPSRC, SHEFC and National Institutes for Health for financial support and the EPSRC equipment loan pool (A. Walker and P. Anthony) for use of the high-speed imaging systems. We also gratefully acknowledge advice and assistance from V. Zarnitzyn and M. Postema (sonoporation), D. McLean (technical construction), M. McDonald (optical trapping) and J. Christophe Bourdon and P. Robertson (cell culture).

Correspondence and requests for materials should be addressed to P.C.

Supplementary Information accompanies this paper on www.nature.com/naturephysics.

\section{Competing financial interests}

The authors declare competing financial interests: details accompany the paper on www.nature.com/naturephysics.

Reprints and permission information is available online at http://npg.nature.com/reprintsandpermissions/ 